Research Article

\title{
Reduced-Complexity Receiver for Free-Space Optical Communication over Orbital Angular Momentum Partial-Pattern Modes
}

\author{
Alaa ElHelaly (D), Ahmed H. Mehana $\mathbb{D}^{D}$, and Mohammad M. Khairy \\ Department of Electronics and Electrical Communications Engineering (EECE), Cairo University, Cairo, Egypt \\ Correspondence should be addressed to Ahmed H. Mehana; ahesham@ieee.org
}

Received 29 December 2017; Revised 10 April 2018; Accepted 15 April 2018; Published 22 May 2018

Academic Editor: Giancarlo C. Righini

Copyright (C) 2018 Alaa ElHelaly et al. This is an open access article distributed under the Creative Commons Attribution License, which permits unrestricted use, distribution, and reproduction in any medium, provided the original work is properly cited.

\begin{abstract}
This paper explores the effect of a partial-pattern receiver for transmitted orbital angular momentum (OAM) multimodes included in the Laguerre-Gaussian beam propagating under non-Kolomogorov weak-to-moderate turbulence on the achievable capacity and the error rates with introduced controlled parameters. We deduce the necessary conditions for reducing the receiver's area to guarantee that the modes are decoupled when the area is reduced. Furthermore, we derive the conditions at which area reduction yields a performance gain over the complete-area reception. For that, some use cases are introduced and discussed and the basic building block for multibeam MIMO receivers with a reduced area is developed and analyzed.
\end{abstract}

\section{Introduction}

The vast majority of the research work exploring orbital angular momentum (OAM) as a mean of free-space optical (FSO) communication has been based on experimental demonstrations [1], turbulence modeling [2-4], MIMO implementations [5], detection enhancement [6], OAM generation $[7,8]$ and capacity derivations $[3,9]$. Maximizing achievable rate by multiplexing vortex beams carrying gigabit streams is also done through space-time coding [10], vortex mode encoding [11, 12], multiple-beam processing [13] or LDPC coding [14].

However, the uniqueness of OAM has not been totally explored in terms of the distinction of the space pattern of each mode. Analogous to filters being deployed to suppress signal interference in both time and frequency domains, this paper introduces small steps in space filtering to decrease the modes interference in a multimode beam communication.
Starting with the reshaping of the basic area, we show that the receiving area can be reduced to special shapes that allow complete mode detection with minimum interference from adjacent modes. Some effort was done in this regard in [15-17] parallel to the presented work where proof of the partial-reception concept is verified. However, this was done through an experiment in only the angular direction while the analytical proof was not done in the presence of either noise or intermode interference.

The motivation behind this work is summarized as follows: First, using high-order modes enlarges the receiver size which is directly proportional to the square root of the OAM mode number [7, 18]; this constrains the maximum number of modes that can be used for a given receiver size. This suggests finding a way to reduce the receiver size upon reception. Second, the propagation of multiple modes in a turbulent medium causes intermode interference. The complexity of the optical devices, which are used to split the modes, grows proportional to 
the number of combined modes. This motivates spatially reshaping the receiver and finding a space-filtering rule to (1) reduce the receiver complexity and (2) to minimize the intermode power leakage. Finally, the simulation time (necessary for any design process) is also proportional to the receiver area; therefore, reducing the beam-matching area cuts down the simulation time while maintaining the same performance results.

To summarize, in this work, we derive the necessary conditions that guarantee the best distinction of the multiplexed OAM modes in a partial-pattern receiver (in both radial and angular directions). We answer the following questions: Is it possible to reduce the receiver area when detecting an OAM signal? What are the conditions that guarantee a similar performance (or better) compared to the full-area receiver? How are the main modes and the intermode leakage affected in the reduced-area receiver?

Our analysis along with Monte Carlo simulations shows that it is fairly possible to reduce the receiver area with either a controlled-performance penalty or performance gain. The system parameters that affect the receiving performance upon cutting the area are: the turbulence strength and the intermodal interference in single and multibeam operations. We also present use cases that exemplify the potential gain of the proposed partial-pattern receiver. Among these use cases, a simplified receiver is proposed with neither power splitting nor additional collimating devices.

The paper is organized as follows. Section 2 provides the communication model. Section 3 presents a partial-pattern receiving concept and derives operational rules. Section 4 derives the signal-to-interference plus noise power ratio for both full- and reduced-plane receivers and derives operational rules. Section 5 discusses cases of single- and multiple-beam-configuration uses. Section 6 combines multipartial patterns and proposes a novel simple-receiver structure. The conclusion is provided in Section 7.

\section{System Model}

Consider a Laguerre-Gaussian (LG) beam carrying a single mode that has the parameters defined in $[19,20]$ with a zero-order Laguerre function carrying the OAM mode " $m$ " affected by the turbulence which has a model defined in [21]. Assume that the beam is carrying the data signal denoted by $S_{m}(t)$ over this single mode " $m$." The noisefree-received waveform (denoted by $U_{m}$ ) for a single mode is written as

$$
\begin{aligned}
U_{m}= & S_{m}(t) \sqrt{\frac{2}{\pi w(z)^{2}(|m| !)}}\left(\frac{\sqrt{2 r}}{w(z)}\right)^{m} e^{\left(r^{2}\right) / w(z)^{2}} e^{i m \theta} e^{i(k z-\omega t)} \\
& \cdot e^{i k(r 2) / 2 R_{c}(z)} e^{-i \varphi(z)} e^{\psi}
\end{aligned}
$$

When this single beam carries multiple modes, the received signal is

$$
y=\sum_{i=m(1)}^{m(M)} \sqrt{\frac{E_{s}}{\mathrm{MP}_{i}}} U_{i} S_{i}=h^{T} S(t),
$$

where we use $h^{T}=\left[h_{1}, h_{2}, \ldots, h_{M}\right]=\sqrt{E_{S} / \mathrm{MP}_{1}}\left[U_{1}, U_{2}, \ldots\right.$, $\left.U_{M}\right], S=\left[S_{1}, S_{2}, \ldots, S_{M}\right]$ after dropping the time variable " $t$ " for simplicity. $R_{c}(z)=z+z_{R}^{2} / z$ is the curvature radius and $w_{0}$ is the beam width at the transmitter, whereas at the receiver we have $w(z)=w_{0} \sqrt{1+\left(z / z_{R}\right)^{2}} \cdot z_{R}=w_{0}^{2} \pi / \lambda$ is the Rayleigh range, $\varphi(z)$ is the Gouy phase, $\varphi(z)=\arctan \left(z / z_{R}\right)$, and $\lambda$ is the beam wavelength. $E_{s i}$ is the symbol's average energy for data carried on mode " $i$ " and $P_{i}$ is the power of mode " $i$ " (we assume all the modes have equal power). Using the above format, each mode's power equals to unity $P_{i}=1$. $M$ is the actual number of the utilized modes $\{m(1), \ldots$, $m(M)\}$.

The exponential term $e^{\psi}$ represents the first-order Rytov approximation for the turbulence induced by the channel. It is generally a complex random variable that is approximated by the lognormal distribution in weak to moderate turbulence $[2,20]$. The term $e^{i m \theta}$ represents the orbital momentum for mode " $m$ " and this is the term that makes the waveforms for different $(m)$ mutually orthogonal $[4,18]$.

At the receiver side, the beam is power divided into $M$ branches, where $M$ is the actual number of used modes $\left\{m_{1}, m_{2}, \ldots, m_{M}\right\}$. Each branch is then matched with a one-beam mode $[1,22,23]$. Ideally, in a turbulence-free environment, only the target mode is received. However, in the presence of the turbulence, all the receiver mode branches output nonzero power; these outputs represent the leakage power from the target transmit mode to all other modes. The matching output power for the target mode relative to the leaked power from other modes is reduced as the turbulence strength increases. The channel estimation is performed by sending a pilot single-mode beam and correlating with $e^{-i n \theta}$ [18] (for every mode $n$ ) followed by an integration process over the receiver area [4].

Let $N_{s}$ and $N_{t}$ be the shot and thermal noise with variances $\sigma_{s}^{2}$ and $\sigma_{n}^{2}$, respectively, as described in [24]. Let $N_{s j}$ and $N_{t j}$ be the corresponding noise terms for the receiver branch " $j$." The output of the mode " $n$ " correlator for the transmit mode " $m$ " is

$$
\begin{aligned}
\overline{y_{m n}} & =\iint_{0,0}^{R, 2 \pi}\left(U_{m}+N_{s}\right) e^{-i n \theta} r d r d \theta \\
& =\sqrt{\left|P_{m n}\right|} S_{m}+\bar{N}_{s} .
\end{aligned}
$$

$P_{m n}$ is the leaked power from the transmit mode $m$ to mode $n$. The matrix with coefficients $\left[P_{m n}\right]$ is the channel efficiency matrix as described in [4]. The overall received signal at branch $j$ denoted by $\left(y_{j T}\right)$ and the data estimation $\widehat{s}_{j T}$ are, respectively, given by

$$
\begin{aligned}
& y_{j T}=\iint_{0,0}^{r 1,2 \pi} e^{-i m_{j} \theta}\left(y+N_{s j}\right) r d r d \theta, \\
& \widehat{s_{j T}}=\int_{0}^{T} S_{j}^{*}\left(y_{j T}+N_{t j}\right) d t .
\end{aligned}
$$




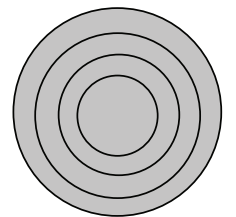

Normal full-power pattern of 3 OAM multiplexed modes

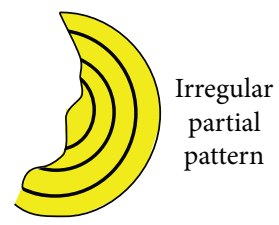

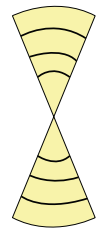

Cones

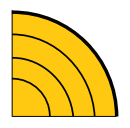

Quarter

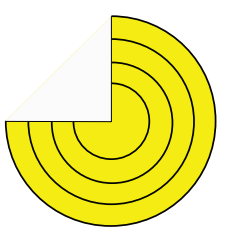

3/4 plane

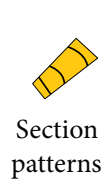

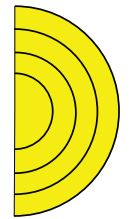

Half

plane

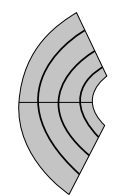

FIGURE 1: Scenarios for reducing the receiving pattern.

The aggregate capacity of the multimode beam is hence given by [9]

$$
\begin{aligned}
C & =\sum_{i} \log _{2}\left(1+\operatorname{SINR}_{i}\right) \\
& =\sum_{i=1}^{r} \log _{2}\left\{1+\frac{E_{s i}\left|h_{i}\right|^{2}}{\sum_{n \neq i} E_{s n}\left|h_{n}\right|^{2}+\sigma_{n}^{2}}\right\} \\
& =\sum_{i=m(1)}^{m(M)} \log _{2}\left(1+\frac{p_{i i}^{M}}{\left\{\sum_{j=m(1), \quad j \neq i}^{m(M)} p_{j i}^{M}\right\}+\sigma_{N m}^{2}}\right) .
\end{aligned}
$$

A superscript " $M$ " is added to $P_{m n}$ to indicate that we are using a multimode beam with " $M$ " number of modes.

\section{Receiver Pattern Area Reduction}

We begin this section by briefly explaining the design process, and then we proceed with the detailed analysis.

In order to design the reduced-pattern receiver, we use (3) with modified integration limits to derive the output of matching the incident beam carrying mode " $m$ " with a partial pattern of mode " $n$." This output is then compared to the output of matching the same beam with a full pattern of mode " $n$." Evaluating the integration in (3) for the partial pattern is analytically intractable in its general form as will be shown.

Alternatively, we provide a bounding technique that allows for a simplified, yet accurate form for the matched filtering output range. This enables a direct comparison between the partial-pattern and the full-pattern matching receivers for both the desired $(m=n)$ and undesired $(m \neq n)$ modes.

We then proceed with the design rules for the reducedarea receiver as follows. We first deduce the necessary conditions in order to obtain the best performance when using a reduced matching area in the absence of the noise and the interference using the derived matching output bounds. This gives good insights of the system parameters and some of the key performance indicators. Finally, we deduce the necessary conditions in the presence of multimode interference and noise. We also provide use cases that show the performance gain and the complexity reduction in single and multibeam operations.

We have a wide range of options while choosing the partial pattern to analyze. Figure 1 shows seven different scenarios: half, quarter, section, and so on. We can even use a hybrid of configurations in a single matching irregular pattern. Our goal is to study how to reduce the receiver area while keeping a controlled impact on the end performance.

As (3) is inspected, the matching area reduction can be done in both angular and radial directions. Starting with a simple analysis, the reduction is performed using a regular shape rather than an irregular shape although this might not be the optimum scenario (cf. Figure 1).

Along the angular direction, the sinusoidal pattern in (1) indicates that each mode has a sine-wave period along that direction. Hence, each mode is uniquely identified upon matching with several cycles of its wave period which does not necessarily cover $2 \pi$ cycle. This suggests the potential use of reducing the matching angular span in possible complete distinction between modes. This comes at the penalty of reducing the collected matching output power which will be overcome in Section 4.

Along the radial direction, there is effectively no added benefit from integrating over areas of almost null values outside the ring of the target mode. This equivalently suggests confining the radial integration limits of (3) to cover the areas where the matching with the target mode yields the large output, whereas the matching with the undesired modes yields small output values. Therefore, the proposed receiver correlates over an area with the limits $0 \leq R_{1} \leq r \leq R_{2} \leq R$ in the radial direction and $0 \leq \theta \leq 2 \pi / N \leq 2 \pi$ in the angular direction for a perfectly aligned receiver (where $R$ is the receiver radius and $N$ is an integer dividing the receiver's area). The area confined between $r=R_{1}$ and $r=R_{2}$ constitutes $98 \%$ of the mode's power. We denote " $N$ " as the "area divisor" as it divides the receiver area by " $N$ " in the angular direction when we use $R_{1}=0$ and $R_{2}=R$. Following the approach in $[4,21]$, starting with the modifying limits of (3) 


$$
\overline{y_{m n}}=\iint_{R_{1}, 0}^{R_{2}, 2 \pi / N}\left(U_{m}+N_{s}\right) e^{-i n \theta} r d r d \theta=\sqrt{\left|P_{m n}\right|} S_{m}+\bar{N}_{s} .
$$

The output power of the partial-pattern matching receiver branch " $n$ " due to the transmit mode " $m$ " is

$$
P_{m n}=\int_{R_{1}}^{R_{2}}\left\langle\left|a_{m n}\right|^{2}\right\rangle r d r
$$

where $a_{m n}$ is the result of matching received mode " $m$ " with branch " $n$ " over the angular dimension. This is the same procedure as in [21] but with limited angular span (from 0 to $2 \pi / N$ rather than from 0 to $2 \pi$ ).

$$
a_{m n}=\frac{1}{\sqrt{2 \pi / N}} \int_{0}^{2 \pi / N} U_{m} e^{-i n \theta} d \theta
$$

and therefore

$$
\begin{aligned}
\left\langle\left|a_{m n}\right|^{2}\right\rangle= & a_{m n} a_{m n}^{*} \\
= & \frac{E_{s}}{(2 \pi / N) M} A_{m}^{2}\left(\frac{2 r^{2}}{\omega^{2}}\right)^{m} e^{\left(-2 r^{2} / \omega^{2}\right)-2 A r^{2}} \int_{0}^{2 \pi / N} e^{-i\left[(m-n) \theta_{2}\right]} \\
& \cdot\left\{\int_{0}^{2 \pi / N} e^{i\left[(m-n) \theta_{1}\right]} e^{2 A r^{2} \cos \left(\theta_{1}-\theta_{2}\right)} d \theta_{1}\right\} d \theta_{2},
\end{aligned}
$$

where $A_{m}=2 / \sqrt{\pi w^{2}|m| !}$

The term $e^{2 A r^{2} \cos \left(\theta_{1}-\theta_{2}\right)}$ in (9) is always a positive quantity and we can approximate (9) (details are in Appendix) to

$$
\begin{aligned}
&\left\langle\left|a_{m n}\right|^{2}\right\rangle \approx \frac{E_{s}}{(2 \pi / N) M} A_{m}^{2}\left(\frac{2 r^{2}}{\omega^{2}}\right)^{m} e^{\left(-2 r^{2} / \omega^{2}\right)-2 A r^{2}} \\
& \cdot\left[e^{2 A r^{2} \cos (2 \pi / N)}+e^{2 A r^{2}}\right]\left[\frac{1-\cos [(m-n)(2 \pi / N)]}{(m-n)^{2}}\right], \\
&\left\langle\left|a_{m n}\right|^{2}\right\rangle \sim \frac{\left[\mathrm{e}^{2 A r^{2}([\cos (2 \pi / N)]-1)}+1\right]}{(2 \pi / N)}\left[\frac{1-\cos [(\mathrm{m}-\mathrm{n})(2 \pi / N)]}{(\mathrm{m}-\mathrm{n})^{2}}\right] .
\end{aligned}
$$

This approximation is simple enough to compare the outputs of partial- and full-pattern matching receivers and proves that reducing the receiving area is possible with some controlled penalty (a rigorous proof is provided in the sequel). From (10) and (11), we conclude the following to guarantee the best achieved performance when using a reduced area.

3.1. Angle Span. In order to differentiate between modes " $m$ " and " $n$ " at the receiver, we equate (11) to zero, therefore

$$
(m-n)=k N \text {, }
$$

where $k$ is an arbitrary integer which implies that the intermode space is a multiple of the area divisor " $N$ ". Condition (12) guarantees the best distinction between modes upon using a partial-pattern receiver.
Comparing full- $\left(N=1 ;\left\langle\left|a_{m n}\right|^{2}\right\rangle_{A}\right)$ and partial-pattern matching $\left(N>1 ;\left\langle\left|a_{m n}\right|^{2}\right\rangle_{P}\right)$ from (10) to (11), we get the following conditions.

For the target mode, $m=n,\left\langle\left|a_{m n}\right|^{2}\right\rangle_{A}$ and $\left\langle\left|a_{m n}\right|^{2}\right\rangle_{P}$ are in the order of

$$
\left\langle\left|a_{m n}\right|^{2}\right\rangle_{A} \sim 2 \pi
$$

and

$\frac{\pi\left[e^{-4 A r^{2}}+1\right]}{N} \leq\left\langle\left|a_{m n}\right|^{2}\right\rangle_{P} \approx \frac{\pi}{N}\left[e^{2 A r^{2}([\cos (2 \pi / N)]-1)}+1\right] \leq \frac{2 \pi}{N}$,

which shows that a reduction in the matching output power is at least by an order of " $N$ " for the target mode.

For the other matching branches, $n \neq m,\left\langle\left|a_{m n}\right|^{2}\right\rangle_{A}$ and $\left\langle\left|a_{m n}\right|^{2}\right\rangle_{P}$ are in the order of

$$
\left\langle\left|a_{m n}\right|^{2}\right\rangle_{A} \sim 0
$$

and

$$
\begin{aligned}
\left\langle\left|a_{m n}\right|^{2}\right\rangle_{P} & \approx \frac{\left[e^{2 A r^{2}([\cos (2 \pi / N)]-1)}+1\right]}{(2 \pi / N)}\left[\frac{1-\cos [(m-n)(2 \pi / N)]}{(m-n)^{2}}\right] \\
& \leq\left\langle\left|a_{m n}\right|^{2}\right\rangle_{P} \leq \frac{N}{\pi}\left[\frac{1-\cos [(m-n)(2 \pi / N)]}{(m-n)^{2}}\right] .
\end{aligned}
$$

The lower limit on the left hand side of the inequality in (16) for the matching branch output represents the output that has the minimum leakage from mode " $m$ " to mode " $n$ " (this is achieved if (12) is satisfied as deduced from (10) and (11)). Whereas, the upper limit on the right hand side is the output power and represents an additional leakage power from mode " $m$ " to mode " $n$ " if (12) is not satisfied.

In general, if (11) is satisfied, there will be no additional leakage power between the modes and the only effect is reducing the target mode output power by a factor of " $N$ ". On the other hand, if (12) is not satisfied, a multiplicative factor to $\left\langle\left|a_{m n}\right|^{2}\right\rangle$ will have its maximum value of $\left[(N / \pi)\left(1-\cos [(m-n)(2 \pi / N)] /(m-n)^{2}\right)\right]$. This is affecting the value of the overall leakage power from the modes beside the turbulence effect. Therefore, we need to satisfy (12) while applying the proposed partial-pattern receiver to avoid this additional leakage power.

3.2. Radial Span. To exclude the null areas outside the mode's ring from the integration in (3), the inner and outer radii of each mode (denoted by $R_{1}$ and $R_{2}$, resp.) can be calculated numerically; however, as a rule of thumb, one can use $R_{1}=0.2 w_{z} \sqrt{l}$ and $R_{2}=2 w_{z} \sqrt{l}$, where $l$ is the mode number, and $w_{z}$ is the beam width at the receiver. This guarantees that over $98 \%$ of the mode's power is included in the obtained matching power. Table 1 shows the exact inner and outer radii that constitute $98 \%$ of the mode's power versus the approximate ones for few modes propagating $1 \mathrm{~km}$ 
TABLE 1: Exact and approximate radii of modes.

\begin{tabular}{|c|c|c|c|c|c|c|c|c|}
\hline \multirow[b]{2}{*}{ Mode number $(m)$} & \multicolumn{4}{|c|}{$R_{1}$} & \multicolumn{4}{|c|}{$R_{2}$} \\
\hline & 1 & 3 & 6 & 14 & 1 & 3 & 6 & 14 \\
\hline $\begin{array}{l}\text { Approximate inner and outer radii constituting more than } 98 \% \text { of a } \\
\text { mode's power (meter) }\left(R_{1}=0.2 w \sqrt{m}, R_{2}=2 w \sqrt{m}\right)\end{array}$ & 0.005 & 0.01 & 0.01 & 0.02 & 0.05 & 0.08 & 0.1 & 0.17 \\
\hline Exact inner and outer radii constitute $98 \%$ of a mode's power (meter) & 0.005 & 0.01 & 0.023 & 0.04 & 0.039 & 0.02 & 0.059 & 0.08 \\
\hline
\end{tabular}
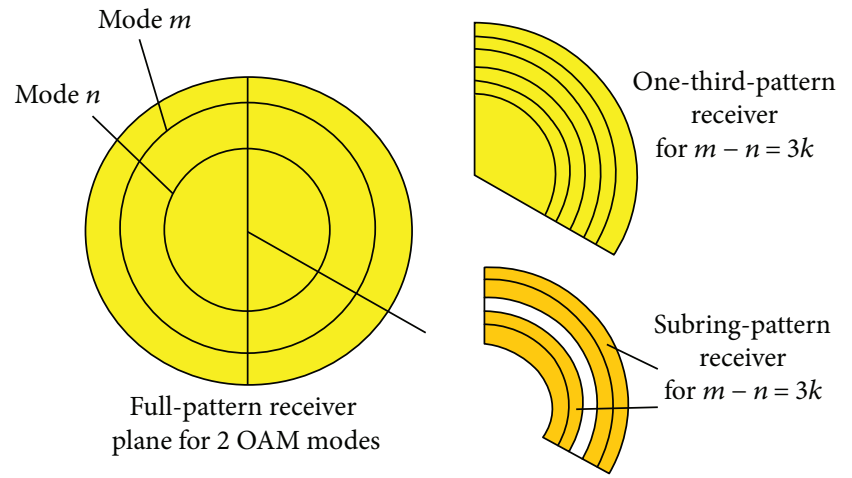

FIGURE 2: One-third subring matching pattern.

over a wavelength of $632.8 \mathrm{~nm}$. The exact mode's power is computed by getting the power of the waveform in (1) for the target mode and integrating over the whole space. To get the exact radii, we iteratively integrate over the radial direction till we reach $98 \%$ of the mode's total power. The limits of integration in (1) would be

$$
0 \leq\left[R_{1} \approx 0.2 w_{z} \sqrt{l}\right] \leq r \leq\left[R_{2} \approx 2 w_{z} \sqrt{l}\right] \leq R .
$$

Figure 2 specifically shows a one-third plane receiver $(N=3)$ and shows an example of a ring receiver covering only each mode's ring, even if it is only part of the ring and not all of it.

It is important to note that the approximation in (10) is used to compare the performance of the original fullpattern receiver with the proposed partial-pattern receiver, and it does not aim at getting the absolute value, and therefore, we are not concerned with getting the approximation error.

\section{Multimode Gain Rules with Reduced- Area Receiver}

We derive here the conditions that guarantee the performance gain of the reduced-pattern receiver in the presence of noise and modes' leakage power. Our reference is the signal to interference plus noise power ratio (SINR) at the receiving end. For the conventional full-pattern receiver for $M$ multiple modes $\left(m_{1}, m_{2} \ldots m_{M}\right)$, SINR for mode " $j$ " can be written as

$$
\operatorname{SINR}_{T}^{j}=\frac{P_{s T}^{j}}{P_{I T}+P_{n T}}=\frac{E_{s} P_{j j}}{\sum_{\substack{i=1 \\ i \neq j}}^{M} P_{i j}+\left(\pi R^{2} \sigma_{s}^{2}+\sigma_{n}^{2}\right)}
$$

The SINR for the case of a partial-pattern receiver (reduced by a factor of $N$ in angular direction) is written as

$$
\operatorname{SINR}_{P}^{j}=\frac{\alpha_{j j}^{2} E_{s} P_{j j}}{\sum_{\substack{i=1 \\ i \neq j}}^{M} \alpha_{i j}^{2} P_{i j}+\pi R^{2} \sigma_{s}^{2}+N \sigma_{n}^{2}},
$$

where we assumed that the total shot noise is directly proportional to the area of the receiver as deduced from [24], hence, $\sigma_{s}^{2}$ is the shot noise power per receiver unit area. The term $\sum_{i=1}^{M} P_{i j}$ represents the leakage power from all $i \neq j$

modes to the target mode " $j$ " and it is the interference term. $\alpha_{j i}^{2} / N$ is an introduced ratio defined as

$\frac{\alpha_{j i}^{2}}{N} \equiv \frac{\text { Partial area matching output power (mode } i \text { matching } j \text { ) }}{\text { Full area matching output power (mode } i \text { matching } i \text { ) }}$.

For some $\alpha_{j i}^{2} \leq 1 \forall i \in\{1: M\}$, the quantity $\alpha_{i j}^{2} / N$ describes the decrease in the output power due to receiving area reduction for the matching branch " $i$ " with the target mode " $j$ " (power ratio). This quantity can be computed from (6), (7), and (9) by using $N=1$ for the full-plane receiver and $N>1$ for the partial plane. The decrease in output power is generally less than or equal to " $N$ " (the area reduction ratio). This follows from the inequality in (16) and can be explained by noting that the sinusoidal nature of modes' cross-sectional pattern over the angular direction makes it sensitive to the integration limits especially when integrating over a small number of sine-wave periods; any small change in the integration limits will decrease the integration value.

Also note that the thermal noise is a fixed term that is not affected by the area reduction. Now comparing (18) and (19), we have two scenarios upon decreasing the matching area; when the decrease in interference plus shot noise power terms (denominator) exceeds the decrease in the intended signal power (numerator), then $\operatorname{SINR}_{P}$ exceeds $\operatorname{SINR}_{T}$ and there is a gain on area reduction. Otherwise, when the decrease in interference plus noise power is less than the decrease in the intended signal power, then $\mathrm{SNR}_{T}$ exceeds $\mathrm{SNR}_{P}$ and there is no gain in area reduction.

Therefore, we are targeting the potential advantage of reducing the area through forcing the communication system to an interference-limited system. We have two controllable parameters that have an effect on this regard: (1) noise power; whether thermal or shot noise, they are related to electronic material technologies and their control will not be discussed here and (2) interference due to power leakage between modes either on the same or different beams; this 


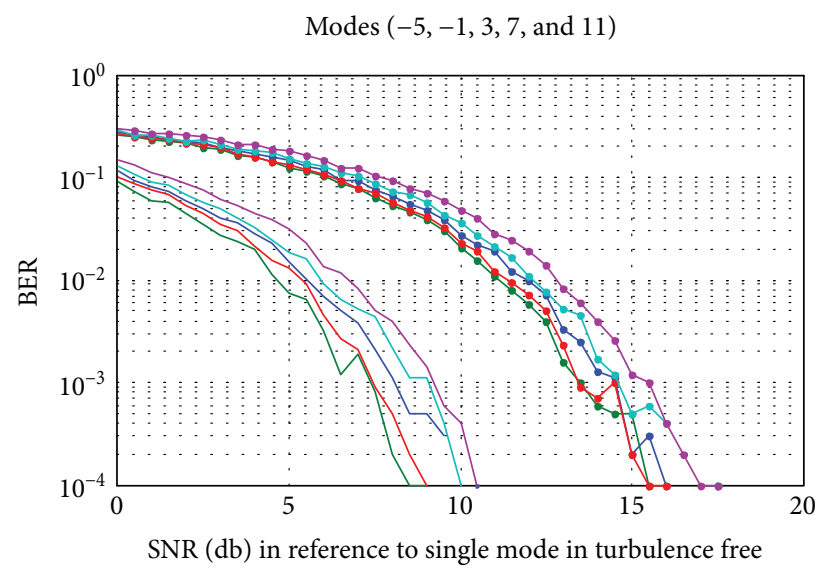

(a)

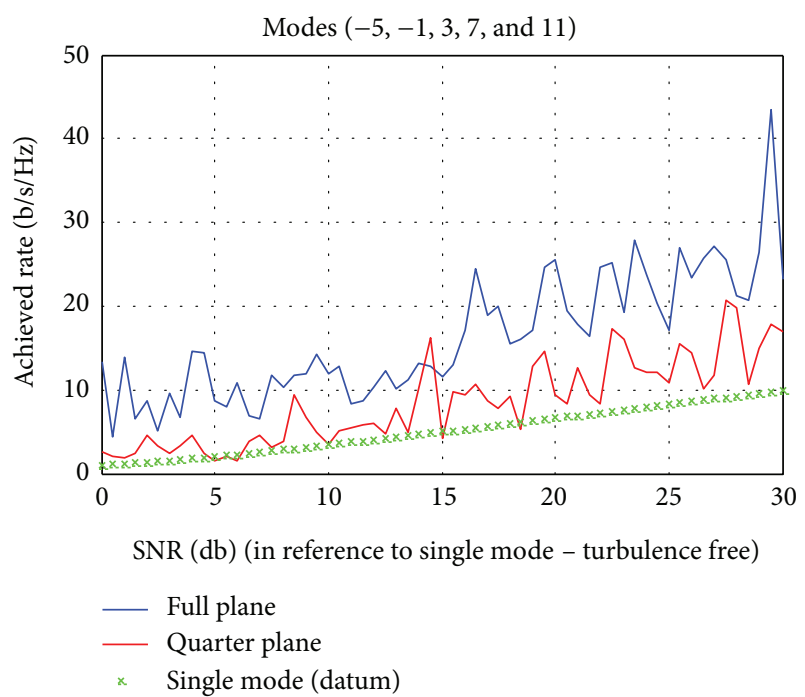

(b)

FIGURE 3: Quarter-area receiver performance: (a) BER performance (6 db loss), dotted lines: quarter-plane receiver and plain lines: full-plane receiver; (b) achieved rates and $\left(C_{n}^{2}=10^{-15}\right), \lambda=\left(632.8 \times 10^{-19}\right) ; L_{0}=1 ; l_{0}=0.01 ; w_{0}=0.02$; propagation $=1 \mathrm{~km}$.

interference can be controlled by selecting the proper mode and controlling the distance between the transmission modes, the distance between the beams in a multibeam system, and the receiver's area reduction scenario which can be one way to force the system to be interference limited. Thus, the gain in the SINR is achieved when (1) interference plus the shot noise dominates over the thermal noise and (2) their decrease due to area reduction is more than the decrease of the intended signal. In other words, from (18) to (19)

$$
\begin{gathered}
\sum_{\substack{i=1 \\
i \neq j}}^{M} \alpha_{i}^{2}\left|a_{\mathrm{i} j}\right|^{2}+\pi R^{2} \sigma_{s}^{2} \gg N \sigma_{n}^{2}, \\
\frac{\alpha_{j}^{2} E_{s}\left|a_{j j}\right|^{2}}{\sum_{\substack{i=1 \\
i \neq j}}^{M} \alpha_{i}^{2}\left|a_{i j}\right|^{2}} \geq \frac{E_{s}\left|a_{j j}\right|^{2}}{\sum_{\substack{i=1 \\
i \neq j}}^{M}\left|a_{i j}\right|^{2}} \forall j \in\{1: M\} .
\end{gathered}
$$

Equivalently, having high-leakage power between the modes due to the high turbulence and possibly high transmission power directs us to design partial-pattern receivers.

When the noise power is dominating the interference (noise-limited system) - violating (21) — we have the normal expected loss as we decrease the receiver area as in Figures 3(a) and 3(b)) which demonstrates the violation of (21) and (22); however, one can still perform a complete mode distinction when the receiver area is reduced as demonstrated in Figure 3 where we are using $N=4$, that is, only 1 quarter of the receiver's area. Yet, as expected, the loss is $6 \mathrm{db}$ (one quarter of the power is lost). This proves that only the proper part of the mode's pattern is sufficient for a complete mode detection. The system of using $N=4$ is equivalent to the system with $N=1$ with a transmitted power reduced by a factor of " $N$."
The loss in power can be compensated as follows by satisfying conditions in (21) and (22).

Conditions (21) and (22) can be satisfied by either carefully selecting the modes that have the highest mutual leakage power or by using several beams with controlled receiving displacements to control the interference power. The following section discusses these two methods.

\section{Reduced-Pattern Performance Gain Use Cases}

5.1. Use Case I: Single-Beam Configuration. Condition (21) can be satisfied when we select transmission modes that have the highest turbulence and these are high modes. Condition (22) is satisfied when the transmission is at high signal power. The maximum performance achieved by the above two scenarios is to have equal performance to the system of complete receiver area or slight gain. However, the performance of data retrieval in this high-turbulence scenario is bad and must be accompanied by further coding or equalization. Therefore, area reduction in this case is beneficial only at low-performing systems when the turbulence effect is strong. Figures 4(a) and 4(b)) show single simulated realization of single-beam transmission with 13 selected modes $(-32,-29,-26,-23,-20,-17,-14,16,19,22,25,28$, and 31) propagating $1 \mathrm{~km}$ over a medium of turbulence index of $C_{n}^{2}=10^{-15}$, a wavelength of $632.8 \mathrm{~nm}$, outer and inner scale turbulence indices of $L_{0}=1$ and $l_{0}=0.01$, respectively, and a transmitter beam width of $0.02 \mathrm{~m}$.

It is shown through Figure 4 that the use of a single beam cannot enable us to have much gain in reducing the receiver's area except with high BER. This motivates us to use area reduction in multiple beams as we discussed in Section 5.2.

5.2. Use Case II: Multiple-Beam Configuration. The conditions imposed by (21) and (22) can be fulfilled by two or 


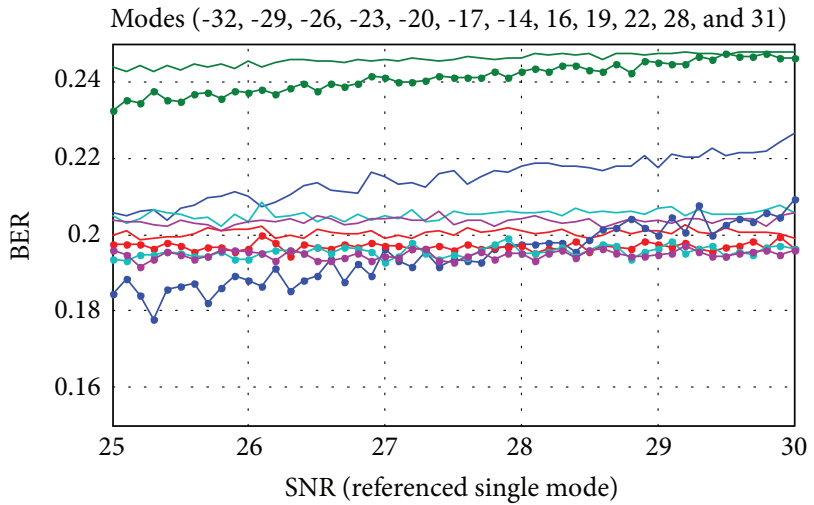

(a)

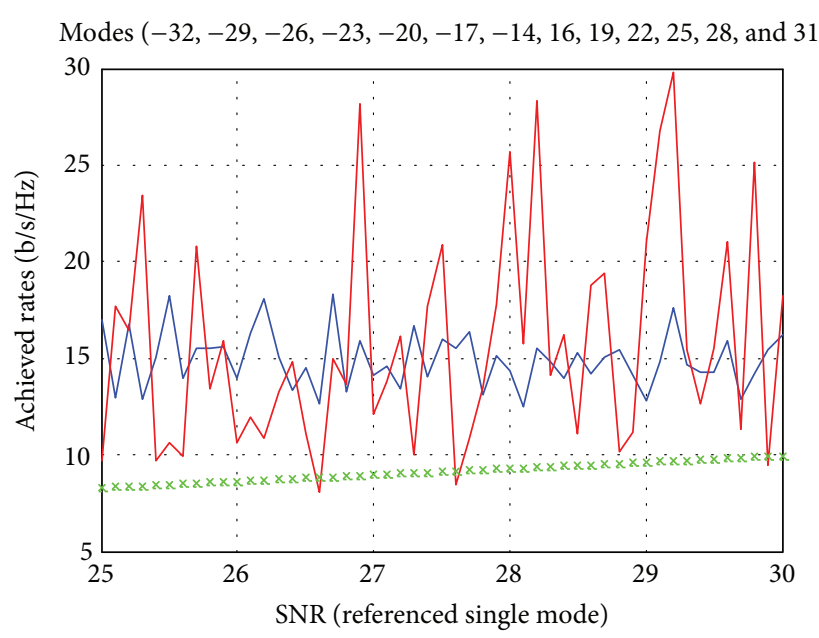

(b)

Figure 4: (a) Selecting high-leakage modes $(-32,-29,-26,-23,-20,-17,-14,16,19,22,25,28$, and 31$)$ and plotting the BER-SNR curve for only five modes. Some modes' performance in the partial-area receiver is better than that of the full plane. Dotted lines: partial plane; plain lines: full plane. Although performance is bad in terms of BER, achieved rates (in Figure 4(b)) are better at some points while using partialarea receivers (same parameters as Figure 3).

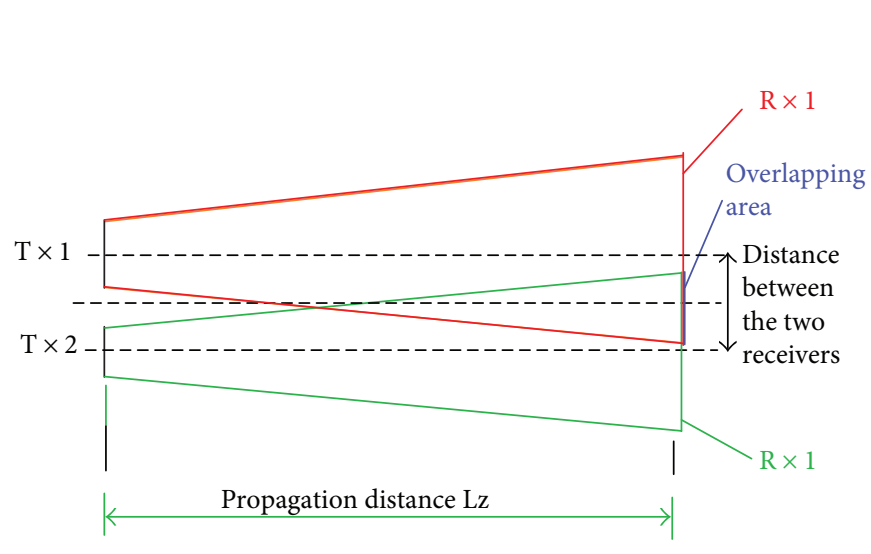

(a)

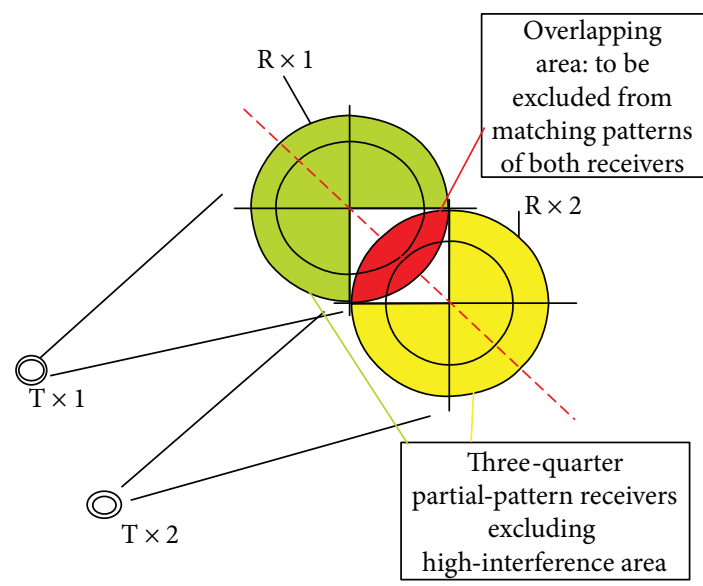

(b)

Figure 5: (a) Two transmitters (Tx1, Tx2) with overlapping beams at the receivers ( $R x 1, R x 2)$. (b) Schematic for two overlapping beams where we exclude the largest interfering quarter of the matching pattern to maximize the achieved rate. Rx1 will be only the green area, and receiver 2 will be the yellow area.

more transmission beams that have overlapping parts at the receiver's side. The largest interference parts would be the core overlapping areas. If we reduce the receiver areas of the two beams to exclude these core overlapping areas, the interference power reduction will be much greater than the intended mode's power reduction. We can have a gain in the achieved rates given that we have a sufficient number of modes.

The receiver configuration is modeled in Figures 5(a) and 5(b)) for two beams having partial overlapping areas with the proposed area reduction to receive over three quarters of the received cross-sectional area. The two receivers' center points are separated by one and a half the radius of the largest mode used.

The resulting achieved rate is shown in Figure 6. The used modes are $(1,2,3,4,5,6,7,8,9,10,11,12,13$, and
14) for both beams. The turbulence index is $C_{n}^{2}=10^{-15}$, wavelength $\lambda=632.8 \mathrm{~nm}$, outer turbulence $L_{0}=10 \mathrm{~m}$, inner scale $l_{0}=0.01 \mathrm{~m}$, transmitter beam width $w_{0}=0.02$, and propagation $=1 \mathrm{~km}$. Notice that the achieved rates of the two reduced-area receivers are better than that of the completearea receivers at a high signal-to-noise power ratio (at SNR greater than $25 \mathrm{db}$ ), and the increase reaches at about $12 \mathrm{~b} /$ $\mathrm{s} / \mathrm{Hz}$ at $\mathrm{SNR}=30 \mathrm{db}$ (about $100 \%$ ); the gap even increases as we increase the transmitted power. Also note that the achieved rate is higher than that of a single receiver with all modes by almost $9 \mathrm{~b} / \mathrm{s} / \mathrm{Hz}$ ( $60 \%$ increase).

Another single realization was done by increasing the overlapping area (two receivers' center points are made apart at the radius of the largest mode used), and two half receivers were used for a further decrease in the receiver total area as in Figure 7. 


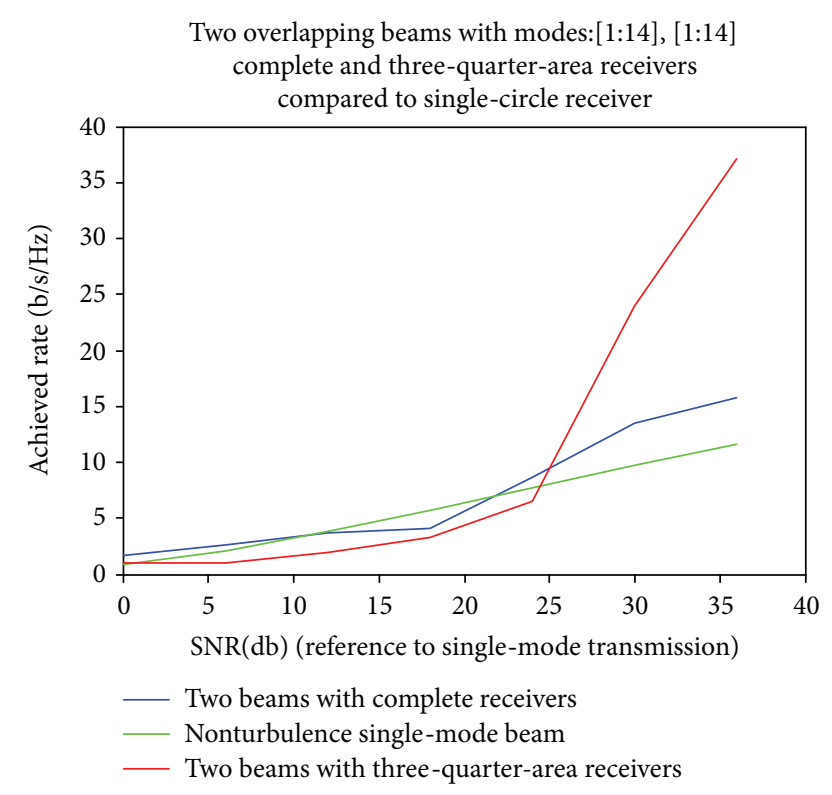

FIGURE 6: $C_{n}{ }^{2}=10^{-15}$, propagation $=1 \mathrm{~km}, L_{0}=10 \mathrm{~m}, l_{0}=1 \mathrm{~cm}$, alpha $=3.1,632.8 \mathrm{~nm}$.

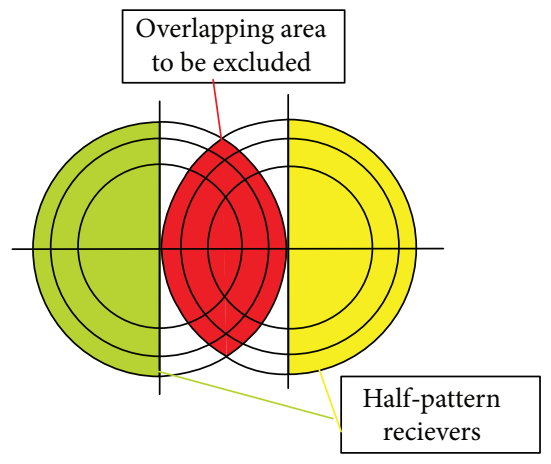

FIgURe 7: Two overlapping beams.

For the configuration in Figure 7, as half-plane receivers were used, the modes' spaces were chosen to be 2 abiding to (12). The outperformance of the half-plane receivers is clear at an SNR greater than $30 \mathrm{~dB}$ with fixed-gap to fullplane receivers by $10 \mathrm{~b} / \mathrm{s} / \mathrm{Hz}$ as shown in Figure 8 .

\section{Partial-Pattern Receiver without Power Splitting}

Let us consider the case of a single beam with four multiplexed modes. A conventional receiver uses three splitting devices with their corresponding collimating devices to have 4 beam copies. Each copy is matched with each of the four modes' pattern to extract the carried data successively (Figure 9(a)). Alternatively, using the proposed partial-pattern receiver, we use a single receiver with no power splitting, but instead we divide the receiver area into four quadrants. Each quadrant is a quarter pattern of one mode and is directly matched to one of the four modes-the four modes are partially matched with the incident beam

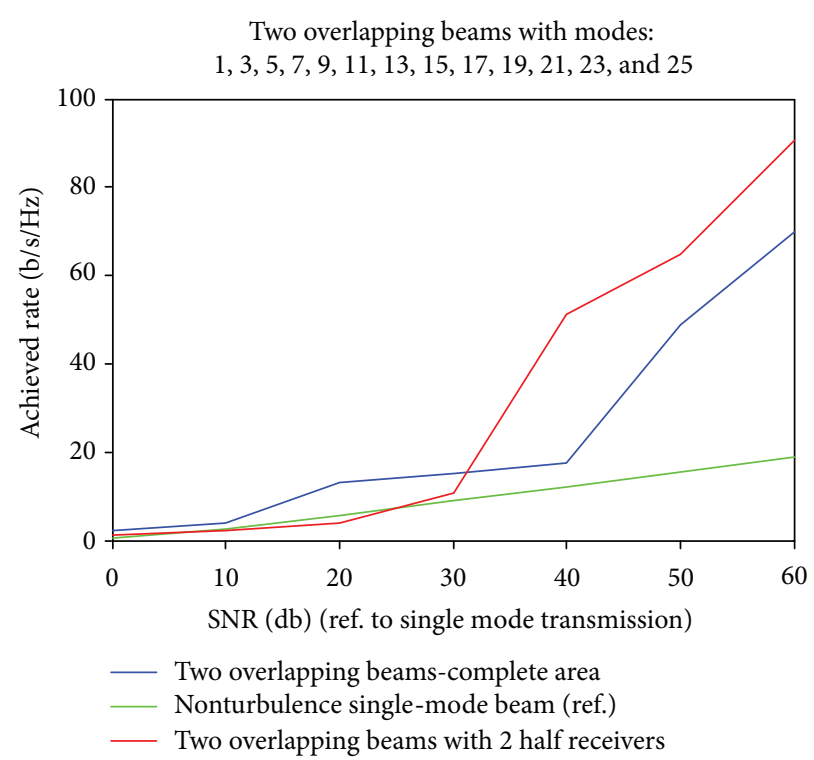

FIgURE 8: $C_{n}{ }^{2}=10^{-15}, 300 \mathrm{~m}, L_{0}=10 \mathrm{~m}, l_{0}=1 \mathrm{~cm}$, alpha $=3.1$, $632.8 \mathrm{~nm}$.

simultaneously. As shown in Section 4 (Figure 3(a)) and the inequality in (12)), the performance of the receiver with a reduced area $(1 / N$ of the conventional area) and transmit power " $P$ " is almost the same as the system with a complete receiver area but with transmitted power equals to $P / N$; this is true when the modes' space is a multiple of " $N$ ". In our example, we can reduce the receiver complexity by a factor of four (due to the savings in the splitting optical devices). In other words, rather than making four complete-area receiver branches along with the power splitters, we can use one full-area receiver without any power splitters and without any other optical devices (that are conventionally used to direct the beams); we can create a matching pattern of each mode in each quarter of this single receiving area. Each quarter would correspond to one receiver branch described in the system model (Sections 2 and 3).

The authors in $[1,22,23]$ describe the different optical elements included in the power division process to create replicas of the main beam at the expense of power loss. Note that the power splitting elements introduce extra losses to include in our comparison. Figure 9 shows a schematic for the conventional receiver in the literature (Figure 9(a)) and the novel simpler one (Figure 9(b)).

Figure 10 shows an example of the combined partialpattern receiver for four modes (1, 4, 8, and 21); Figure 10(a) shows the four complete-pattern receivers versus the combined single partial-pattern one in Figure 10(b). The wavelength $=632.8 \mathrm{~nm}$, initial beam width $=2 \mathrm{~mm}$, and receiver radius $=10 \mathrm{~cm}$.

In general, if we are using $M$ modes and we are dividing the pattern by $N(N=4$ in Figure 9$)$, then the reduction in hardware in the proposed simple receiver with respect to the splitting devices is $M / N$; if we have 12 modes and we are using quarter sectors, then we split the main beam into 3 rather than 12 subbeams and each of the 3 subbeams uses the combined partial-pattern receiver. 


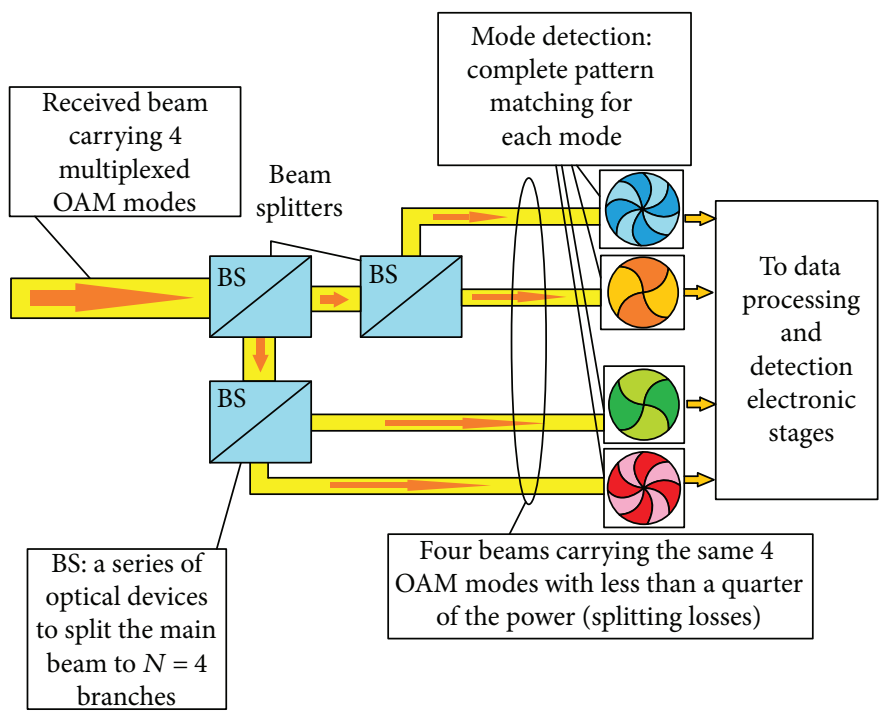

(a)

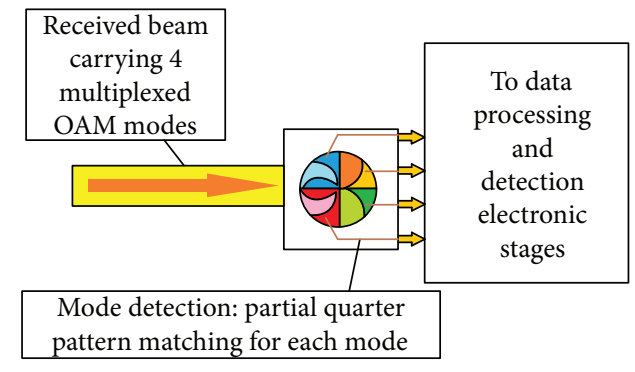

(b)

FIGURE 9: (a) Conventional 4-mode matching receiver with multiple branches. (b) Simplified receiver with four quadrants and no splitting devices.
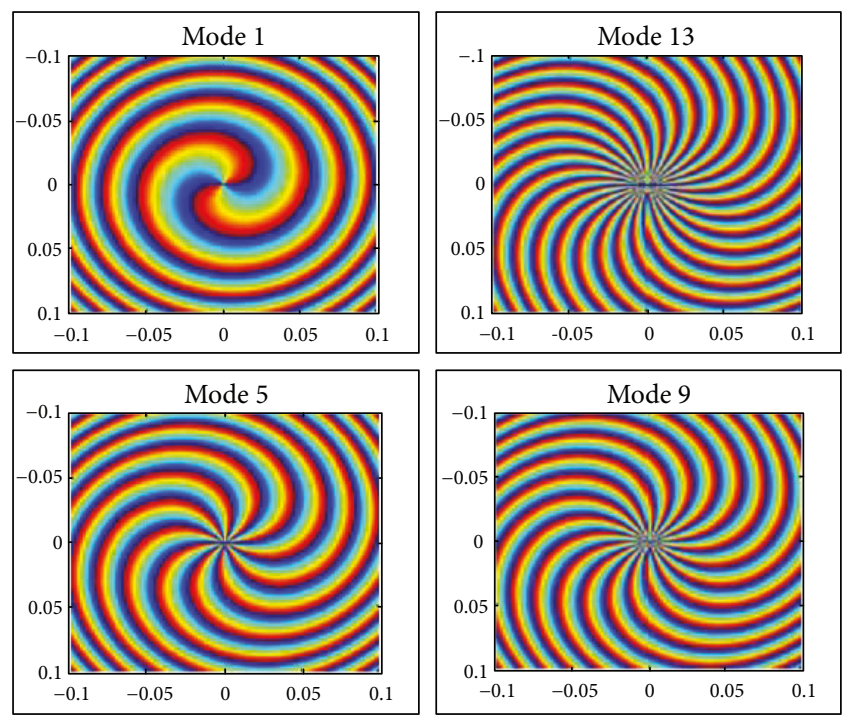

(a)

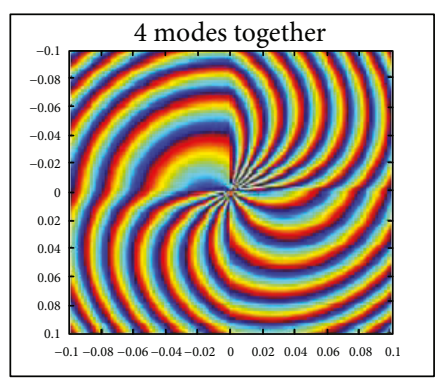

(b)

FIgURE 10: (a) Conventional receiver with four complete matching spiral-phase patterns for modes $(1,5,9$, and 13$)$ versus the combined 4 quarter matching partial patterns in the simple receiver in Figure 10(b).

The simplicity introduced of putting $N$ patterns matching in one receiver plane opens the future for easier implementation of MIMO systems.

\section{Conclusion}

In this paper, we propose a simple receiver architecture for OAM communication based on the partial-pattern receiver. Three rules have been concluded to guarantee a controlled performance impact while decreasing the receiver area using a single beam: (1) In the angular direction, the modes' separation must be multiples of the reduction ratio. (2) In the radial direction, the integration process should be limited to cover the ring range for the target mode. (3) There should be high intermode interference in the medium- to highturbulence environment and high SNR. Carefully selected modes and transmit power can lead to a large performance gain. However, the high potential gain of reducing the receiver area is more effective when using a multibeammultimode configuration. We derived rules to achieve gain while using partial patterns in multiple beams. The achieved rates are verified via simulations at high SNR. The proposed receiver can be further used as a building block for using the MIMO system of overlapping beams carrying multiple OAM modes. Equalizations can also be added in this work for further enhancements. The partial patterns are then combined 
in one pattern receiver to introduce the basic form of a simple receiver where the optical devices are set to a minimum.

\section{Conflicts of Interest}

The authors declare that there are no conflicts of interest regarding the publication of this paper.

\section{Appendix}

\section{Approximation of (10)}

From (9) and looking further to the integral $\left\{\int_{0}^{2 \pi / N} e^{i\left[(m-n) \theta_{1}\right]}\right.$ $\left.e^{2 A r^{2} \cos \left(\theta_{1}-\theta_{2}\right)} d \theta_{1}\right\}$, the term $e^{2 A r^{2} \cos \left(\theta_{1}-\theta_{2}\right)}$ is always a positive quantity with a least value of $e^{-2 A r^{2}}$ and a maximum value of $e^{2 A r^{2}}$, and we safely approximate the integral by replacing the $e^{2 A r^{2} \cos \left(\theta_{1}-\theta_{2}\right)}$ by its arithmetic mean value with respect to $\theta_{1}$ as $1 / 2\left[e^{2 A r^{2} \cos \left((2 \pi / N)-\theta_{2}\right)}+e^{2 A r^{2} \cos \left(\theta_{2}\right)}\right]$, which is similarly a positive term and can be approximated by the mean value with respect to $\theta_{2}$, hence

$$
\begin{aligned}
& \left\langle\left|a_{m n}\right|^{2}\right\rangle \approx \frac{E_{s}}{2 \pi / N} A_{m}^{2}\left(\frac{2 r^{2}}{\omega^{2}}\right)^{m} \mathrm{e}^{\left(-2 r^{2} / \omega^{2}\right)-2 A r^{2}} \int_{0}^{2 \pi / N} \frac{1}{2} \\
& \cdot\left[e^{2 A r^{2} \cos \left((2 \pi / N)-\theta_{2}\right)}+e^{2 A r^{2} \cos \left(\theta_{2}\right)}+\right] \\
& \cdot e^{-i\left[(m-n) \theta_{2}\right]}\left\{\int_{0}^{2 \pi / N} e^{i\left[(m-n) \theta_{1}\right]} d \theta_{1}\right\} d \theta_{2} \\
& \approx \frac{E_{s}}{2 \pi / N} A_{m}^{2}\left(\frac{2 r^{2}}{\omega^{2}}\right)^{m} e^{\left(-2 r^{2} / \omega^{2}\right)-2 A r^{2}} \int_{0}^{2 \pi / N} \frac{1}{4} \\
& \cdot\left[e^{2 A r^{2} \cos (2 \pi / N)}+e^{2 A r^{2} \cos (0)}\right. \\
& \left.+e^{2 A r^{2} \cos ((2 \pi / N)-(2 \pi / N))}+e^{2 A r^{2} \cos (2 \pi / N)}\right] \\
& \cdot e^{-i\left[(m-n) \theta_{2}\right]}\left\{\int_{0}^{2 \pi / N} e^{i\left[(m-n) \theta_{1}\right]} d \theta_{1}\right\} d \theta_{2} \\
& \approx \frac{E_{s}}{2 \pi / N} A_{m}^{2}\left(\frac{2 r^{2}}{\omega^{2}}\right)^{m} e^{\left(-2 r^{2} / \omega^{2}\right)-2 A r^{2}} \frac{1}{2} \\
& \cdot\left[e^{2 A r^{2} \cos (2 \pi / N)}+e^{2 A r^{2}}\right] \int_{0}^{2 \pi / N} e^{-i\left[(m-n) \theta_{2}\right]} \\
& \left\{\int_{0}^{2 \pi / N} e^{i\left[(m-n) \theta_{1}\right]} d \theta_{1}\right\} d \theta_{2} \\
& \approx \frac{E_{s}}{2 \pi / N} A_{m}^{2}\left(\frac{2 r^{2}}{\omega^{2}}\right)^{m} \mathrm{e}^{\left(-2 r^{2} / \omega^{2}\right)-2 A r^{2}} \frac{1}{2} \\
& \cdot\left[e^{2 A r^{2} \cos (2 \pi / N)}+e^{2 A r^{2}}\right]\left[\frac{e^{i[(m-n)(2 \pi / N)]}-1}{i(m-n)}\right] \\
& \cdot\left[\frac{e^{-i[(m-n)(2 \pi / N)]}-1}{-i(m-n)}\right] \\
& \approx \frac{E_{s}}{2 \pi / N} A_{m}^{2}\left(\frac{2 r^{2}}{\omega^{2}}\right)^{m} \mathrm{e}^{\left(-2 r^{2} / \omega^{2}\right)-2 A r^{2}} \frac{1}{2} \\
& \cdot\left[e^{2 A r^{2} \cos (2 \pi / N)}+e^{2 A r^{2}}\right]
\end{aligned}
$$

$$
\begin{aligned}
& \cdot\left[\frac{1-e^{i[(m-n)(2 \pi / N)]}-e^{-i[(m-n)(2 \pi / N)]}+1}{(m-n)^{2}}\right] \\
\approx & \frac{E_{s}}{2 \pi / N} A_{m}^{2}\left(\frac{2 r^{2}}{\omega^{2}}\right)^{m} \mathrm{e}^{\left(-2 r^{2} / \omega^{2}\right)-2 A r^{2}} \\
& \cdot\left[e^{2 A r^{2} \cos (2 \pi / N)}+e^{2 A r^{2}}\right] \\
& \cdot\left[\frac{1-\cos [(m-n)(2 \pi / N)]}{(m-n)^{2}}\right],
\end{aligned}
$$

which concludes (10).

\section{References}

[1] Y. Ren, Z. Wang, G. Xie et al., "Demonstration of OAM-based MIMO FSO link using spatial diversity and MIMO equalization for turbulence mitigation," in Optical Fiber Communication Conference, IEEE, Anaheim, CA, USA, 2016, Electronic ISBN: 978-1-9435-8007-1.

[2] B. Rodenburg, M. Mirhosseini, M. Malik et al., "Simulating thick atmospheric turbulence in the lab with application to orbital angular momentum communication," New Journal of Physics, vol. 16, no. 3, article 033020, 2014.

[3] Y. Zhu, X. Liu, J. Gao, Y. Zhang, and F. Zhao, "Probability density of the orbital angular momentum mode of Hankel-Bessel beams in an atmospheric turbulence," Optics Express, vol. 22, no. 7, pp. 7765-7772, 2014.

[4] J. A. Anguita, M. A. Neifeld, and B. V. Vasic, "Turbulenceinduced channel crosstalk in an orbital angular momentummultiplexed free-space optical link," Applied Optics, vol. 47, no. 13, pp. 2414-2429, 2008.

[5] Y. Ren, Z. Wang, G. Xie et al., "Free-space optical communications using orbital-angular-momentum multiplexing combined with MIMO-based spatial multiplexing," Optics Letters, vol. 40, no. 18, pp. 4210-4213, 2015.

[6] G. Xie, Y. Ren, H. Huang et al., "Phase correction for a distorted orbital angular momentum beam using a Zernike polynomials-based stochastic-parallel-gradient-descent algorithm," Optics Letters, vol. 40, no. 7, pp. 1197-1200, 2015.

[7] J. Kupferman and S. Arnon, "Receiver design for OWC orbital angular momentum communication in data center applications," in 2016 10th International Symposium on Communication Systems, Networks and Digital Signal Processing (CSNDSP), pp. 1-6, Prague, Czech Republic, 2016.

[8] L. Cheng, W. Hong, and Z.-C. Hao, "Generation of electromagnetic waves with arbitrary orbital angular momentum modes," Scientific Reports, vol. 4, no. 1, p. 4814, 2014.

[9] A. Paulraj, Introduction to Space-Time Wireless Communication, Cambridge University Press, New York City, NY, USA, 2008.

[10] Y. Zhang, P. Wang, L. Guo, W. Wang, and H. Tian, "Performance analysis of an OAM multiplexing-based MIMO FSO system over atmospheric turbulence using space-time coding with channel estimation," Optics Express, vol. 25, no. 17, pp. 19995-20011, 2017.

[11] J. Kupferman and S. Arnon, "Decoding Algorithm for Vortex Communications Receiver," Journal of Optics IOP Publishing $L t d$, vol. 20, no. 1, 2017. 
[12] X. Ge, R. Zi, X. Xiong, Q. Li, and L. Wang, "Millimeter wave communications with OAM-SM scheme for future mobile networks," IEEE Journal on Selected Areas in Communications, vol. 35, no. 9, pp. 2163-2177, 2017.

[13] S. Li and J. Wang, "Experimental demonstration of optical interconnects exploiting orbital angular momentum array," Optics Express, vol. 25, no. 18, pp. 21537-21547, 2017.

[14] Z. Qu and I. B. Djordjevic, " $500 \mathrm{~Gb} / \mathrm{s}$ free-space optical transmission over strong atmospheric turbulence channels," Optics Letters, vol. 41, no. 14, pp. 3285-3288, 2016.

[15] Y. Hu, S. Zheng, X. Jin et al., "Simulation of orbital angular momentum radio communication systems based on partial aperture sampling receiving scheme," IET Microwaves, Antennas \& Propagation, vol. 10, no. 10, pp. 1043-1047, 2016.

[16] J. Clement, B. A. Vourch, and T. D. Drysdale, "Planar millimetre-wave antenna simultaneously producing four orbital angular momentum modes and associated multielement receiver array," IET Microwaves, Antennas \& Propagation, vol. 10, no. 14, pp. 1492-1499, 2016.

[17] S. Zheng, X. Hui, J. Zhu et al., "Orbital angular momentum mode-demultiplexing scheme with partial angular receiving aperture," Optics Express, vol. 23, no. 9, pp. 12251-12257, 2015.

[18] J. Wang, J.-Y. Yang, I. M. Fazal et al., “Terabit free-space data transmission employing orbital angular momentum multiplexing," Nature Photonics, p. 488, 2012.

[19] X. Sheng, Y. Zhang, X. Wang, Z. Wang, and Y. Zhu, “The effects of non-Kolmogorov turbulence on the orbital angular momentum of a photon-beam propagation in a slant channel," Optical and Quantum Electronics, vol. 43, pp. 121-127, 2012.

[20] L. C. Andrews and R. L. Phillips, Laser Beam Propagation through Random Media, SPIE-Press, Bellingham, WA, USA, 2nd edition, 2005.

[21] Y. S. Jiang, S. H. Wang, J. H. Zhang, J. Ou, and H. Tang, "Spiral spectrum of Laguerre-Gaussian beam propagation in nonKolmogorov turbulence," Optics Communications, vol. 303, pp. 38-41, 2013.

[22] Y. Yan, G. Xie, M. P. J. Lavery et al., "High-capacity millimetre-wave communications with orbital angular momentum multiplexing," Nature Communications, vol. 5, 2014.

[23] I. B. Djordjevic and M. Arabaci, "LDPC-coded orbital angular momentum (OAM) modulation for free-space optical communication," Optics Express, vol. 18, no. 24, article 24722, p. $24728,2010$.

[24] F. Laforce, "Low noise optical receiver using Si APD," in Proceedings Volume 7212, Optical Components and Materials VI, San Jose CA, USA, 2009. 


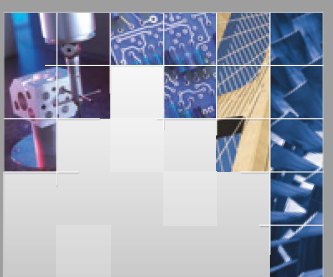

\section{Enfincering}
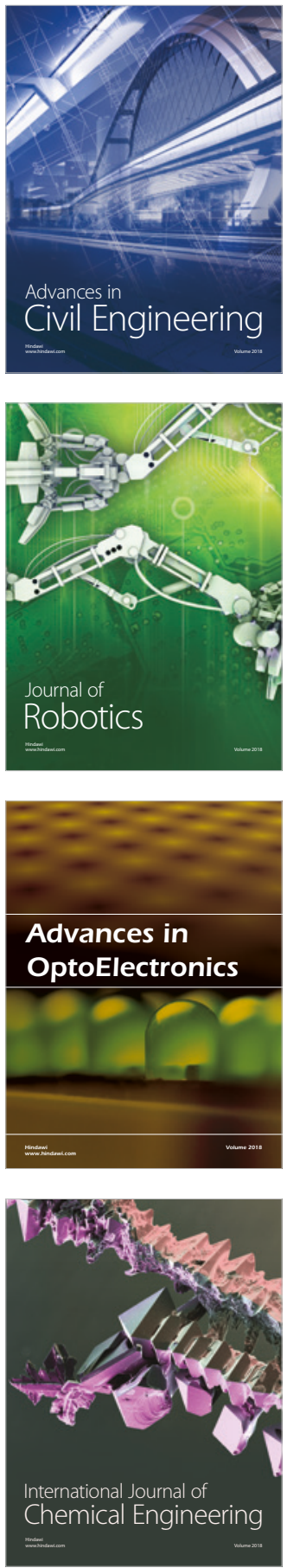

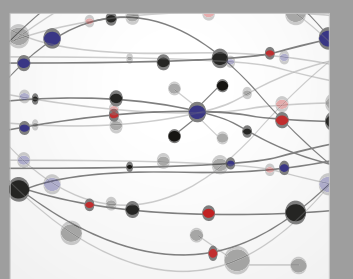

\section{Rotating \\ Machinery}

The Scientific World Journal

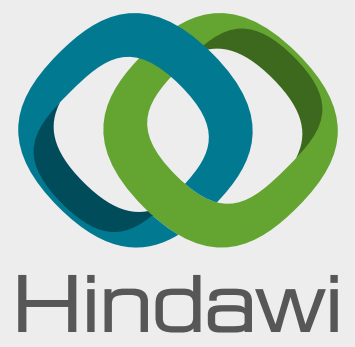

Submit your manuscripts at

www.hindawi.com
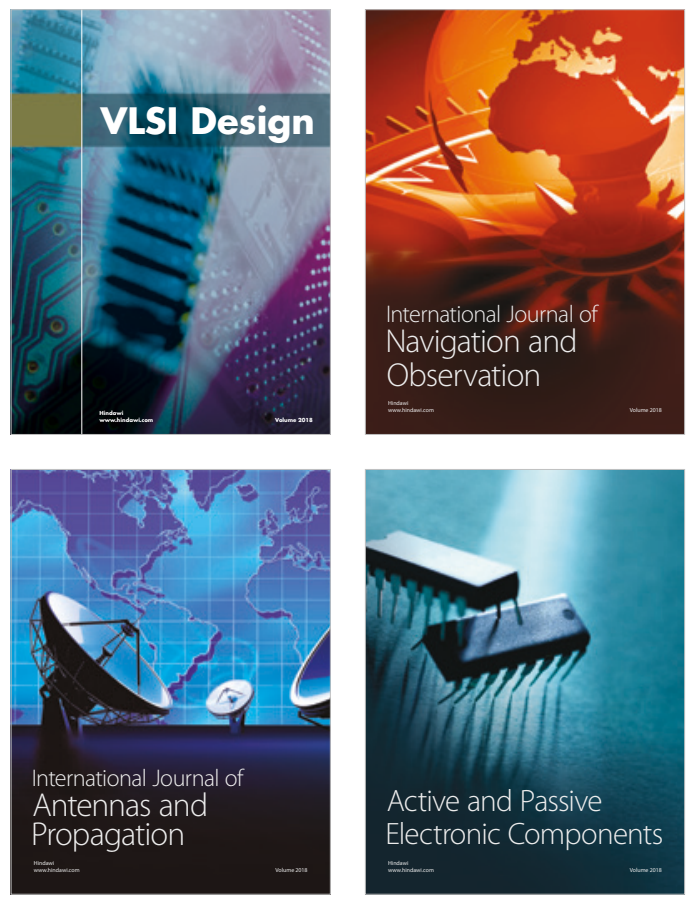
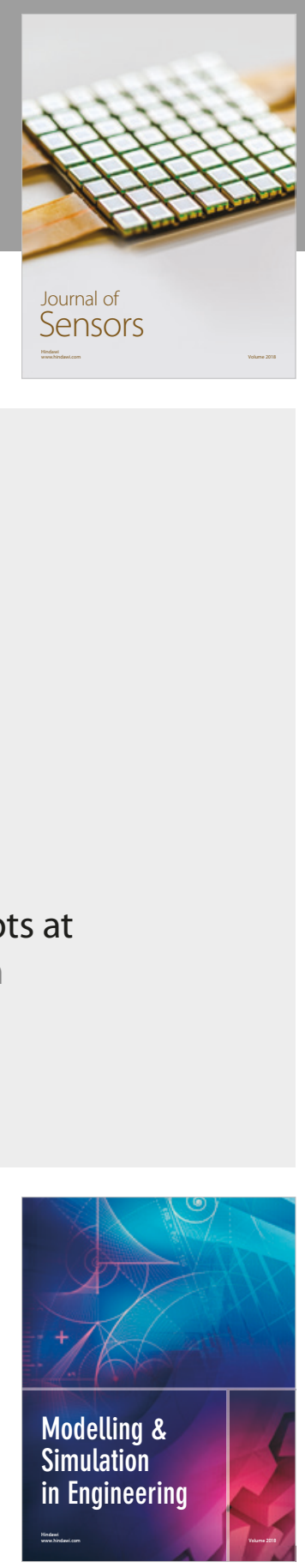

\section{Advances \\ Multimedia}
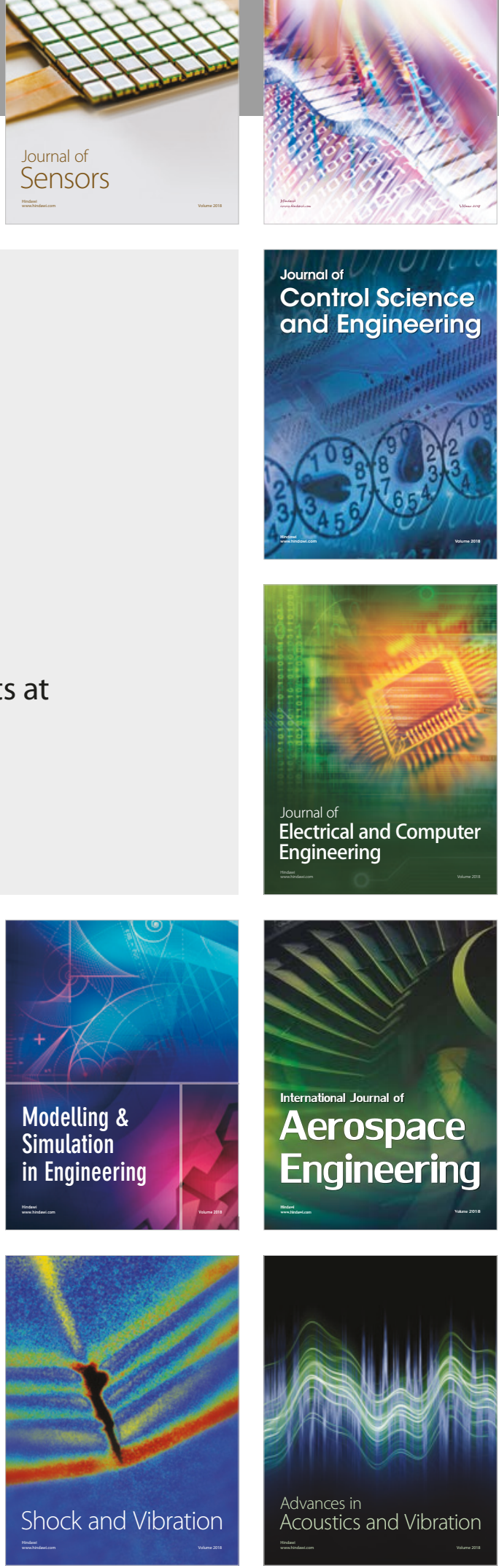